\title{
Zero-Order and Prolonged Release of Atenolol from Microporous FAU and BEA Zeolites, and Mesoporous MCM-41: Experimental and Theoretical Investigations
}

\author{
A.J. Wise ${ }^{1}$, J Sobhani Sefy ${ }^{1}$, E. Barbu ${ }^{1}$, A.J. O'Malley ${ }^{2}$, S.M. van der Merwe ${ }^{1}$ and P.A. Cox ${ }^{1, *}$ \\ *Corresponding author: E-mail address: paul.cox@port.ac.uk, Telephone +44 2392-843577 \\ ${ }^{1}$ School of Pharmacy and Biomedical Sciences, University of Portsmouth, Portsmouth, UK. \\ ${ }^{2}$ The Centre for Sustainable and Circular Technologies (CSCT), Department of Chemistry, University of Bath, \\ Claverton Down, Bath, BA2 7AY
}

Word count for Abstract: 146 words

Complete manuscript word count: 4664

Number of references: 34

Number of figures: 6

Number of tables: 2

Declarations of interest: none

This research did not receive any specific grant from funding agencies in the public, commercial, or not-for-profit sectors. 


\section{Abstract}

The potential of microporous zeolites FAU and BEA, and mesoporous MCM-41, for prolonged release of atenolol in drug delivery systems was investigated both experimentally, using drug release studies, and theoretically using classical molecular dynamics simulations.

Remarkably, zero-order release of atenolol was achieved from $\mathrm{FAU}\left(\mathrm{SiO}_{2} / \mathrm{Al}_{2} \mathrm{O}_{3}=80: 1\right)$ into phosphate buffer for 24 hours followed by prolonged release for at least another 48 hours. Experimental data also demonstrate the ability for all of the drug-zeolite combinations investigated to achieve prolonged release of atenolol, with the release rates determined by the combination of framework topology, aluminium content and drug release study media.

Molecular dynamics simulations give an insight into the reasons for the different release rates observed for FAU and BEA. The results of this work emphasise the need for sophisticated models in order to model subtle differences in release, such as those observed at different $\mathrm{SiO}_{2} / \mathrm{Al}_{2} \mathrm{O}_{3}$ ratios.

Keywords:

Zeolite

Atenolol

Controlled Release

Zero-order

Drug Release

Molecular Dynamics 


\section{Introduction}

The potential of various inorganic materials as carriers for drugs has been investigated with a view to improving efficacy or reducing side-effects. Various ordered microporous and mesoporous silica structures have been the subject of recent interest including zeolites $(1,2)$ and mesoporous materials, such as MCM-41 $(3,4)$.

Zeolites are microporous crystalline materials with well-defined structures made from interconnecting $\mathrm{SiO}_{4}$ and $\mathrm{AlO}_{4}{ }^{-}$ tetrahedra. They have many properties which make them good candidates for drug delivery systems including having a unique microporosity, large surface area, large pore volume, narrow pore size distribution, good biocompatibility and low toxicity. There are over 150 known distinct structures of zeolites each containing interconnecting pores of $0.2-2 \mathrm{~nm}$ diameter and channels of various dimensions that can be large enough to accommodate drug molecules of different structures and sizes.

Zeolites can be produced such that aluminium atoms are included in the framework in place of a proportion of the silicon atoms. The aluminium content is described by the silica: alumina $\left(\mathrm{SiO}_{2}: \mathrm{Al}_{2} \mathrm{O}_{3}\right)$ ratio i.e. a ratio of $360: 1$ indicates that there are 180 silicon atoms for every aluminium atom. Each aluminium atom in the framework results in a negative charge being induced on one of the adjacent oxygen atoms which in turn requires a positively charged counter-ion in order to maintain the neutrality of the framework. Common examples of counter-ions include $\mathrm{H}^{+}, \mathrm{Na}^{+}$ and $\mathrm{NH}_{4}{ }^{+}$. When the counter-ion is hydrogen, Brønsted Acid sites are formed, resulting in a more acidic framework than those with lower aluminium content. Alteration of the ratio of aluminium to silicon atoms can be used to manipulate the properties of the framework, including hydrophobicity/ hydrophilicity, charge and acidity, both within the internal channel system and on the external surface(2,5-9).

It has been shown that, if zeolites are loaded with appropriately selected drug molecules, the release of the drug into dissolution media can be modified in order to increase bioavailability, slow down or speed up the release of a drug $(9,10)$. Examples of zeolites with distinctly different pore, channel and cage systems that have been investigated for use in drug delivery systems include zeolite beta (BEA) and faujasite (FAU). BEA is a 3D system comprised of straight and sinusoidal 12 member ring channels (6.7 $\AA$ in diameter), while FAU is made up from sodalite cages linked by hexagonal prisms to form super cages ( $13 \AA$ in diameter linked by $7 \AA$ windows) (11).

Mesoporous materials (pore sizes of 2-50nm), such as MCM-41, also have the ability to modify drug release rates $(4,12)$. As with zeolites, MCM-41 can be produced with alumino-substitution of a proportion of the silicon atoms in order to manipulate the physicochemical properties of the internal channels and external surface, and MCM-41 therefore shares the desirable physical properties of zeolites. In addition to these properties it is proposed that, as mesoporous materials have a larger pore volume and tuneable pore size, they have the potential to be used as drug reservoirs with a large loading capacity $(4,13)$.

While it is possible to use a combination of drug and zeolite or mesoporous material to produce a modified release drug delivery system, selection of an appropriate combination of framework and drug can be difficult and, in order to optimise host-drug combinations, it is desirable to have a screening mechanism which is able to identify those combinations that are likely to be suitable for experimental testing. Classical Molecular Dynamics (MD) simulations have been proposed as a tool for screening drug-zeolite combinations prior to further experimental investigations $(14,15)$.

In this work, commercially available zeolite and mesoporous frameworks with different topologies and $\mathrm{SiO}_{2}: \mathrm{Al}_{2} \mathrm{O}_{3}$ ratios (table 1) $(11,16-20)$ have been investigated for their potential use in oral prolonged release drug delivery systems for the model drug atenolol. Atenolol was chosen as a model drug because, due to possessing both polar and non-polar regions (figure 1), the interactions with frameworks are difficult to predict. The amount of drug released during these experiments is not intended to reflect a therapeutic dose but instead allow insight into factors that affect the rate of drug release. 
Since all the frameworks being investigated in this study are insoluble in water, the mechanism for modifying the drug release rate is likely to be similar to that of a matrix polymer. Accordingly, the release rate will be determined by the rate of diffusion of the drug molecule through the framework channels or cages. MD uses simulations to model the molecular mobility of the drug molecule within a framework, and comparison of MD simulation results therefore has the potential to predict which drug-framework combinations will release the drug faster or slower in drug release studies. One of the aims of this study is to further evaluate the usefulness of the technique by making comparisons with the observed experimental data for a molecule with such a mixture of hydrophilic and hydrophobic regions.
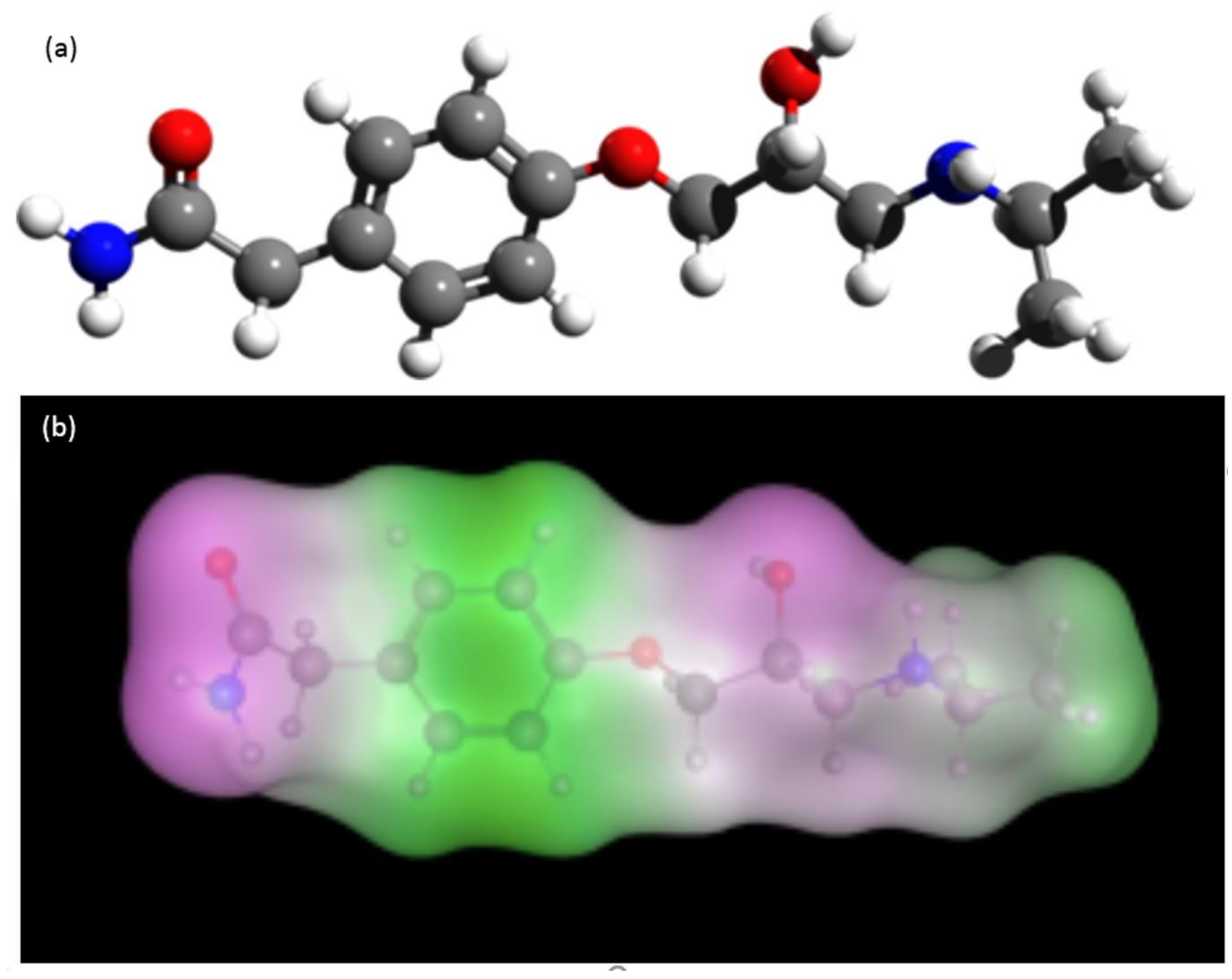

Figure 1 Atenolol (a) ball and stick structure and (b) visualisation of the lipophilic regions in green and hydrophilic regions in purple 


\section{Material and Methods}

\section{Materials}

Zeolites were purchased from Alfa Aesar. Mesoporous materials and atenolol were purchased from Sigma Aldrich. Acetone (laboratory reagent grade) was purchased from Fisher Chemical. Phosphate buffered saline (pH 7.4) and concentrated $\mathrm{HCl}$ was purchased from Fisher Scientific. $\mathrm{HCl}$ was diluted with deionised water to produce a $0.1 \mathrm{M}$ solution. All purchased chemicals were used as received without further purification.

\begin{tabular}{lcccc}
\hline $\begin{array}{c}\text { Zeolite/ Mesoporous } \\
\text { Material }\end{array}$ & $\begin{array}{c}\text { Counter- } \\
\text { Ion }\end{array}$ & $\begin{array}{c}\mathrm{SiO}_{2}: \mathrm{Al}_{2} \mathrm{O}_{3} \\
\text { ratio }\end{array}$ & $\begin{array}{c}\text { The largest diameter of a sphere that } \\
\text { can diffuse along the channels/ } \AA\end{array}$ & $\begin{array}{c}\text { Specific Surface } \\
\text { Area/ } \mathrm{m}^{2} / \mathrm{g}\end{array}$ \\
\hline BEA & $\mathrm{H}^{+}$ & $360: 1$ & 5.95 & 620 \\
FAU & & & 7.35 & 730 \\
& $\mathrm{H}^{+}$ & $5.1: 1$ & 7.35 & 780 \\
MCM-41 & $80: 1$ & $21-27$ & $\sim 1000$ \\
Alumino-MCM-41 & & $\infty$ & & \\
\hline
\end{tabular}

Table 1 Summary of Zeolites investigated (16-20)

\section{Preparation of drug loaded zeolites and mesoporous materials}

$200 \mathrm{mg}$ of zeolite or mesoporous material was soaked for 4 hours at room temperature in a solution of $8 \mathrm{mg}$ atenolol dissolved in $40 \mathrm{~mL}$ acetone. The solvent was then removed by filtration and the solid sample was dried overnight in an oven at $70^{\circ} \mathrm{C}$ before storage in a desiccator prior to analysis.

\section{Thermogravimetric Analysis}

Thermogravimetric analysis (TGA) was carried out using a TG 209 F1 Libra instrument (Netzsch, Germany), with a constant heating rate of $10^{\circ} \mathrm{C} / \mathrm{min}$. Nitrogen $\left(50 \mathrm{~mL} / \mathrm{min}\right.$ flow) was used as inert gas from $25^{\circ} \mathrm{C}$ up to $600^{\circ} \mathrm{C}$, when the carrier was switched to dry air $\left(50 \mathrm{~mL} / \mathrm{min}\right.$ flow; from $\left.600^{\circ} \mathrm{C}-800^{\circ} \mathrm{C}\right)$ to allow for the combustion of the organic residue. Data was processed using Netzsch Proteus v.6.1 analysis software, which was used to include the Derivative Thermogravimetric data (DTG).

\section{Drug Release Studies}

Tablets of the loaded zeolite samples were prepared using a single punch tablet press with a $13 \mathrm{~mm}$ die under a load of 3 tonnes for 30 seconds. The release of atenolol from the tablets was then analysed for 24 hours using USP/ Ph.Eur. basket dissolution apparatus (Pharma Test PT-DT7) to measure absorbance at a wavelength of $225 \mathrm{~nm}$ in $\mathrm{HCl} 0.1 \mathrm{M}$ ('acid'-pH 1.2) and phosphate buffer ('buffer'-pH 7.4). FAU 80:1 samples were analysed for a further 48 hours (total 72 hours) due to drug release extending beyond 24 hours. Calibration curves were plotted to enable absorbance values to be converted into drug concentration and calculation of the mass of drug released. A control sample was also used during each drug release test and the absorbance values of the control were subtracted from the test sample values. All studies were carried out under sink conditions in triplicate and data presented are the average values with standard deviation presented as error bars. All reasonable steps were taken to prevent evaporation during the experiment. 


\section{Molecular dynamics simulations}

Molecular dynamics calculations were undertaken using the program Materials Studio (21). A fully siliceous model was used for the host frameworks, with no constraints applied to the movement of the atoms. A $1 \times 1 \times 1$ supercell of FAU with dimensions $(25.028 \AA \AA$ x $25.028 \AA$ x $25.028 \AA$ ) and a $4 \times 2 \times 1$ supercell of BEA with dimensions of $(50.645 \AA \mathrm{x}$ $25.323 \AA$ $\times 26.406 \AA$ ) were used as the simulation box for the zeolite frameworks. For MCM-41, the model of the disordered hydroxylated structure developed by Ugliengo et al was used (22), with a supercell of dimensions $(40.603 \AA \times 40.603 \AA \times 73.21 \AA$ ) . Periodic boundary conditions were applied with a loading of one molecule per simulation box. Calculations were performed for 20000000 steps with a time step of $1 \mathrm{fs}$, with the atomic coordinates recorded every $20 \mathrm{ps}$. The first $500 \mathrm{ps}$ of the run were used to equilibrate the system and this data was discarded from the subsequent analysis. The CVFF forcefield was used in these simulations. This forcefield was chosen as it has been widely used to successfully to simulate the location of organic molecules within zeolite hosts (see for example reference 23). Ewald summation was used to calculate the electrostatic component of the energy, while a cut-off of $12.5 \AA$ was used for the short-range interactions. Cartesian coordinates of the atenolol centroid were extracted for each recorded frame of the simulation data allowing calculation of the distance travelled between each recorded frame. All simulations were carried out in triplicate and average values are shown in the graphs.

One of the requirements of periodic molecular dynamics calculations is that the overall charge of the system is neutral. For the zeolite systems investigated here, simulations were performed on fully siliceous frameworks where the overall charge on the drug and zeolite were both zero (but with appropriate partial charges calculated for the individual atoms). Simulations were also carried out for a protonated atenolol molecule located within a charged (1) framework. This charge was 'smeared' across all of the framework oxygen atoms using the charge adjustment tool available within Materials Studio. 

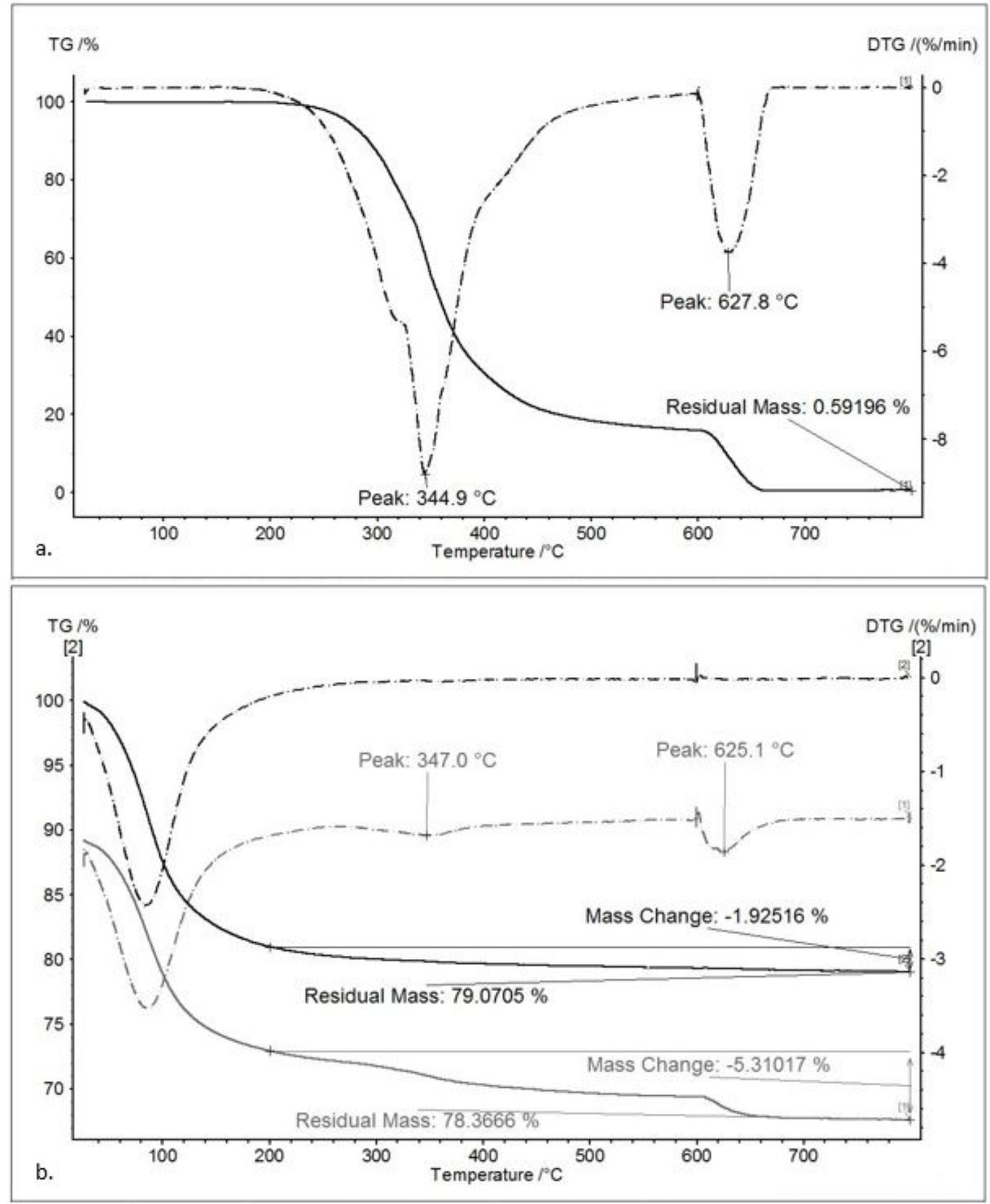

Figure 2 TGA (solid line) and DTG (dashed line) of a. pure atenolol, b. overlaid unloaded FAU 5.1:1 H+ framework (black) and FAU 5.1:1 H+ loaded with atenolol (grey) 


\begin{tabular}{|c|c|c|c|c|c|c|}
\hline \multirow{2}{*}{$\begin{array}{l}\text { Zeolite/ } \\
\text { Mesoporous } \\
\text { Material }\end{array}$} & \multirow{2}{*}{$\begin{array}{l}\text { Estimated Drug } \\
\text { Content of Test } \\
\text { Samples/mg }\end{array}$} & \multirow{2}{*}{$\begin{array}{l}\text { Estimated } \\
\text { Loading } \\
\text { Efficiency }\end{array}$} & \multicolumn{2}{|c|}{$\begin{array}{l}\text { Mass of Atenolol } \\
\text { Released/ mg }\end{array}$} & \multicolumn{2}{|c|}{$\begin{array}{l}\text { Percentage of } \\
\text { Atenolol Released/\% }\end{array}$} \\
\hline & & & Acid & Buffer & Acid & Buffer \\
\hline BEA & 2.25 & $58.5 \%$ & 0.15 & 0.27 & 6.67 & 12.0 \\
\hline FAU 5.1:1 & 3.39 & $88.1 \%$ & 2.68 & 2.00 & 79.1 & 59.0 \\
\hline FAU 80:1 & 3.10 & $80.6 \%$ & 1.84 & 1.56 & 59.4 & 50.3 \\
\hline MCM-41 & 3.10 & $80.6 \%$ & 1.81 & 2.00 & 58.3 & 64.5 \\
\hline Alumino-MCM-41 & 3.83 & $99.5 \%$ & 2.89 & 3.51 & 75.4 & 91.6 \\
\hline
\end{tabular}

Table 2 Summary of Final Experimental Drug Loading and Drug Release Values Summary of Final Experimental Drug Loading and Drug Release Values. 'Acid' is $0.1 \mathrm{M} \mathrm{HCl}$ pH1.2 and 'buffer' is phosphate buffer pH7.4.

Typical TGA results (for pure atenolol, unloaded FAU 5.1:1 $\mathrm{H}^{+}$, and FAU 5.1:1 $\mathrm{H}^{+}$loaded with atenolol) are presented in figure 2. The thermogram of pure atenolol (solid line) shows its degradation starts above $260^{\circ} \mathrm{C}$, with a residual mass at $600^{\circ} \mathrm{C}$ of less than $20 \%$ and followed by the complete combustion of the organic residue.

The data from the unloaded samples (zeolites only) show a mass change step typically around $100^{\circ} \mathrm{C}$, due to the loss of physisorbed water from within the frameworks. However, in addition to the initial loss of water, the unloaded samples appear to continue to lose mass - very slowly - across the entire temperature range of the experiment. This is perhaps not surprising since it is known that zeolites are able to retain water within their frameworks at temperatures above the boiling point of water and this minor mass change step may therefore represent continued water loss throughout the duration of the experiment(24). No subsequent mass loss associated with high temperature combustion was observed after switching to air as a carrier at $600^{\circ} \mathrm{C}$. The unloaded mesoporous materials do not lose as much water as the unloaded zeolite samples ( $2-3 \%$ vs. 8-20\% respectively), reflective of a reduced content of physisorbed water in mesoporous materials compared to zeolites.

The thermograms of the drug loaded samples also show a mass loss step at around $100^{\circ} \mathrm{C}$, similar to the unloaded samples, due to loss of physisorbed water; additionally, at higher temperatures, a mass loss step associated with atenolol degradation can be observed. The loaded drug content was calculated by subtracting the total percentage mass loss values above $200^{\circ} \mathrm{C}$ (mass loss loaded - unloaded).

Table 2 displays the estimated drug content of the drug loaded zeolite test samples based on TGA data as well as the estimated loading efficiency calculated as follows:

$$
\text { Estimated loading efficiency }=\frac{\text { Actual drug content of loaded zeolite calculated from TGA data (\%) }}{3.84(=\text { maximum possible drug content of loaded zeolite }(\%))} \times 100
$$

The data show that the loading of atenolol into the frameworks is dependent on both the topology and the $\mathrm{SiO}_{2}$ : $\mathrm{Al}_{2} \mathrm{O}_{3}$ ratio of the framework, with greatest loading in frameworks with larger pores and greater aluminium content. Some possible explanations for these observations are considered in the discussion. 


\section{Drug Release Studies}

Release Profile of Atenolol from Each Framework into pH $1.2 \mathrm{HCl} 0.1 \mathrm{M}$

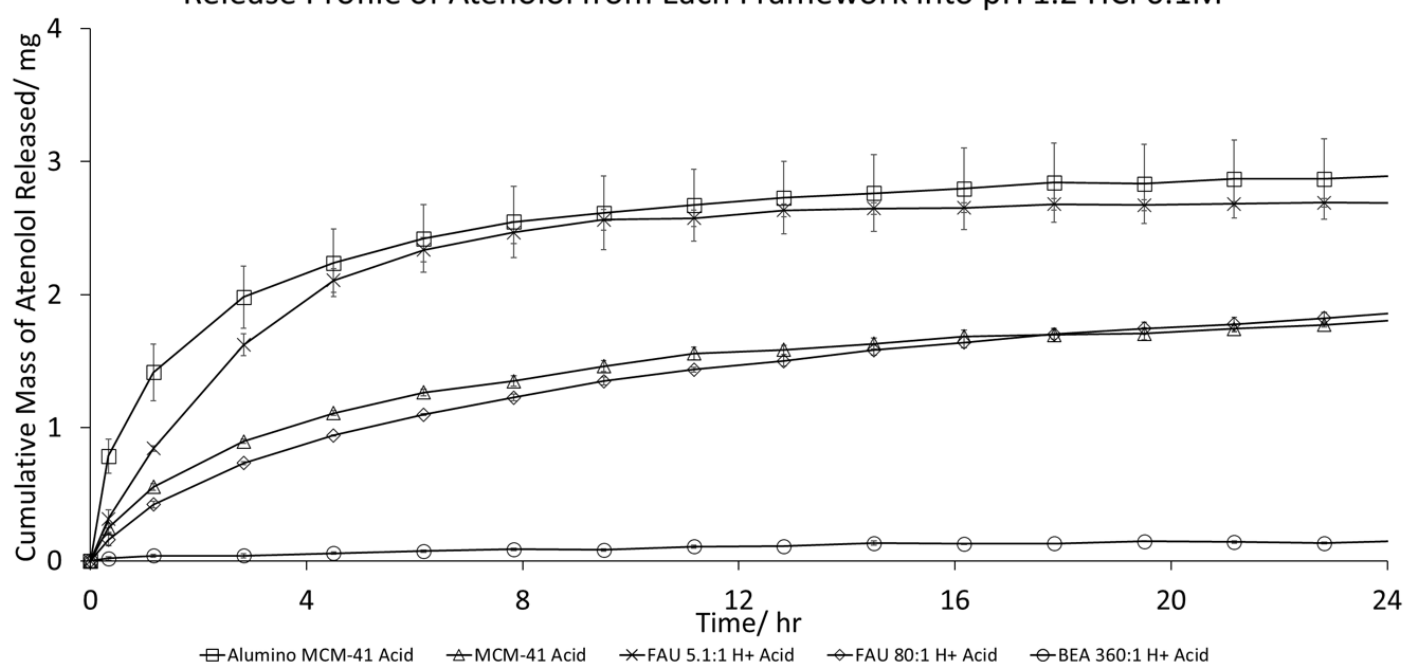

Release Profile of Atenolol from Each Framework into pH 7.4 Phosphate

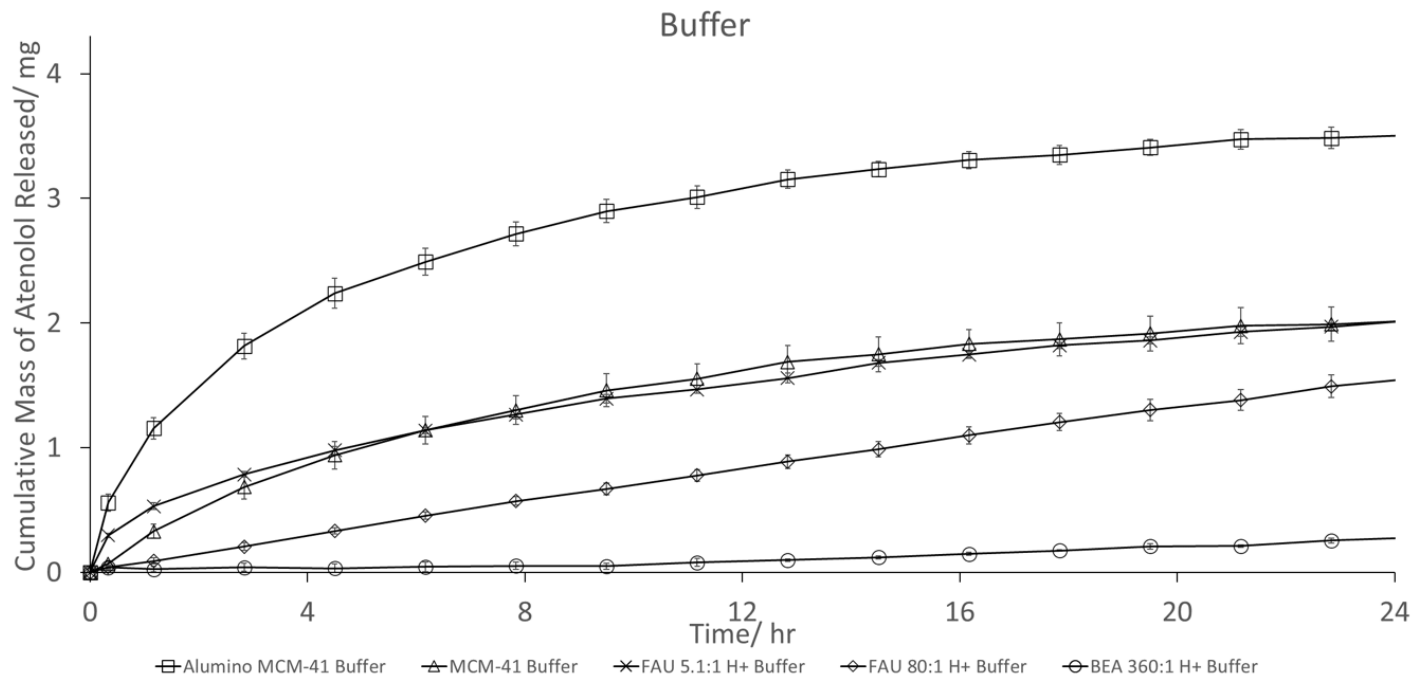

Release of Atenolol from FAU 80:1 over 72 Hours

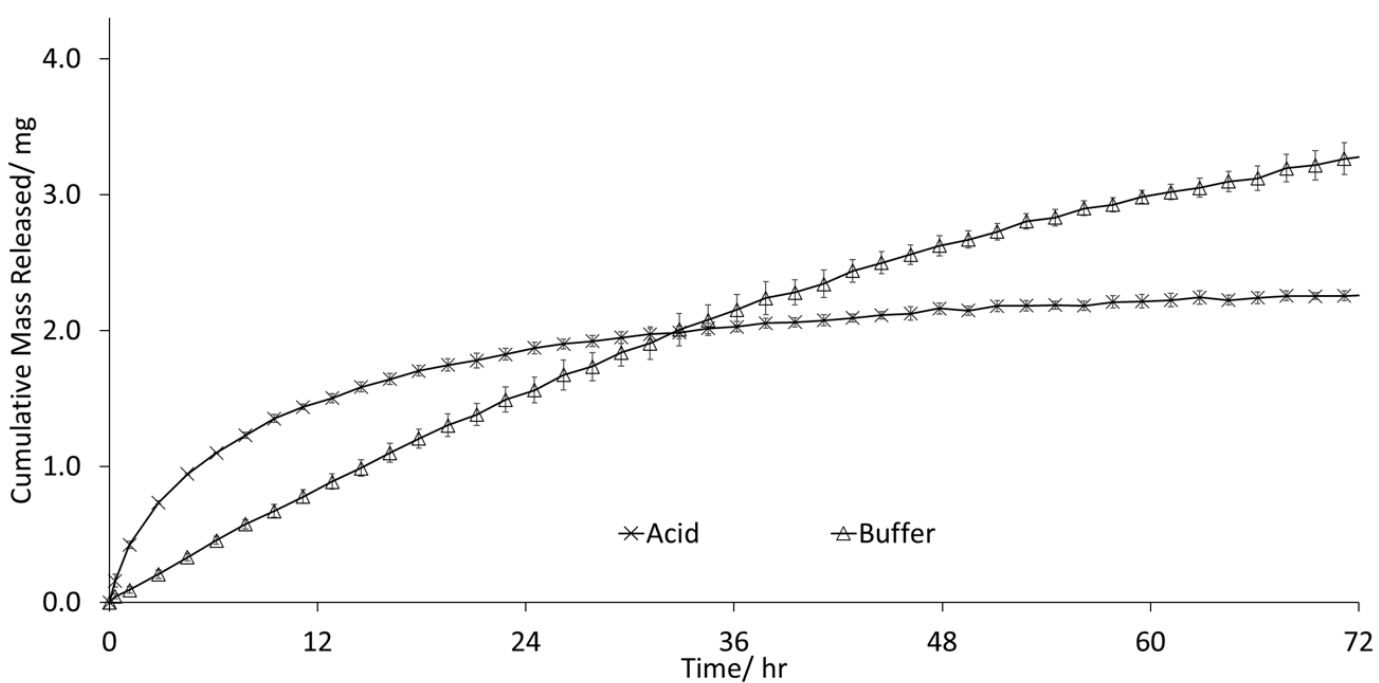

Figure 3 Comparison of Cumulative Release from Each Framework into $\mathrm{HCl} 0.1 \mathrm{M}$ 'Acid' (pH1.2) and Phosphate 'Buffer' (pH7.4). Error bars shown are standard deviation for all graphs NB: some error bars are very small and obscured by the markers 
Table 2 summarises the final mass and percentage release of atenolol tests after 24 hours of drug release studies. Figure 3 shows the drug release profiles of atenolol from the test samples over 24 hours into 'acid' (top) and 'buffer' (middle) as well as the drug release profile of atenolol from FAU 80:1 into both acid and buffer over 72 hours (bottom).

The results for the release of atenolol from FAU 80:1 into the $\mathrm{pH} 7.4$ phosphate buffer demonstrate that if the correct combination of $\mathrm{pH}$, framework topology and $\mathrm{SiO}_{2}: \mathrm{Al}_{2} \mathrm{O}_{3}$ ratio are used, a zero-order modified release drug delivery system can be achieved using a relatively cheap and simple system. The release profile from FAU 80:1 into buffer solution is quite remarkable considering the simplicity of the drug delivery system, with linear $\left(R^{2}=0.9959\right)$ release for 24 hours, and prolonged release continuing for at least 72 hours. Since transdermal patches, and other drug delivery systems, employ the use of a matrix polymer similar to the system investigated here, it is interesting to note that it may be possible to formulate a dosage form for routes other than oral delivery such as transdermal delivery via a patch which could last for at least 72 hours.

The result for FAU 80:1 into $\mathrm{pH} 7.4$ phosphate buffer is in stark contrast to other samples which produce a burst release at the start of the experiment followed by slower release of the drug. Again, the speed, quantity and duration of drug release is determined by the combination of $\mathrm{pH}$, framework topology and $\mathrm{SiO}_{2}: \mathrm{Al}_{2} \mathrm{O}_{3}$ ratio.

These results, although not as remarkable as the FAU 80:1 sample described above, demonstrate the potential of a range of ordered microporous and mesoporous materials to produce prolonged release drug delivery systems that have varying drug release profiles. The potential explanations for the observed results are considered in the discussion.

Also notable from the drug release studies was the observation that the tablets remained intact at the end of the experiment and did not break up, dissolve, or disperse. This is a helpful observation to note as, although it is already known that the frameworks are insoluble in water, dispersal of the tablets into smaller particles would have increased the surface area, introducing another variable that could have affected the drug release rate. Since the tablets are all equal in diameter and of similar thickness, the surface area is roughly comparable for all of the test samples. 

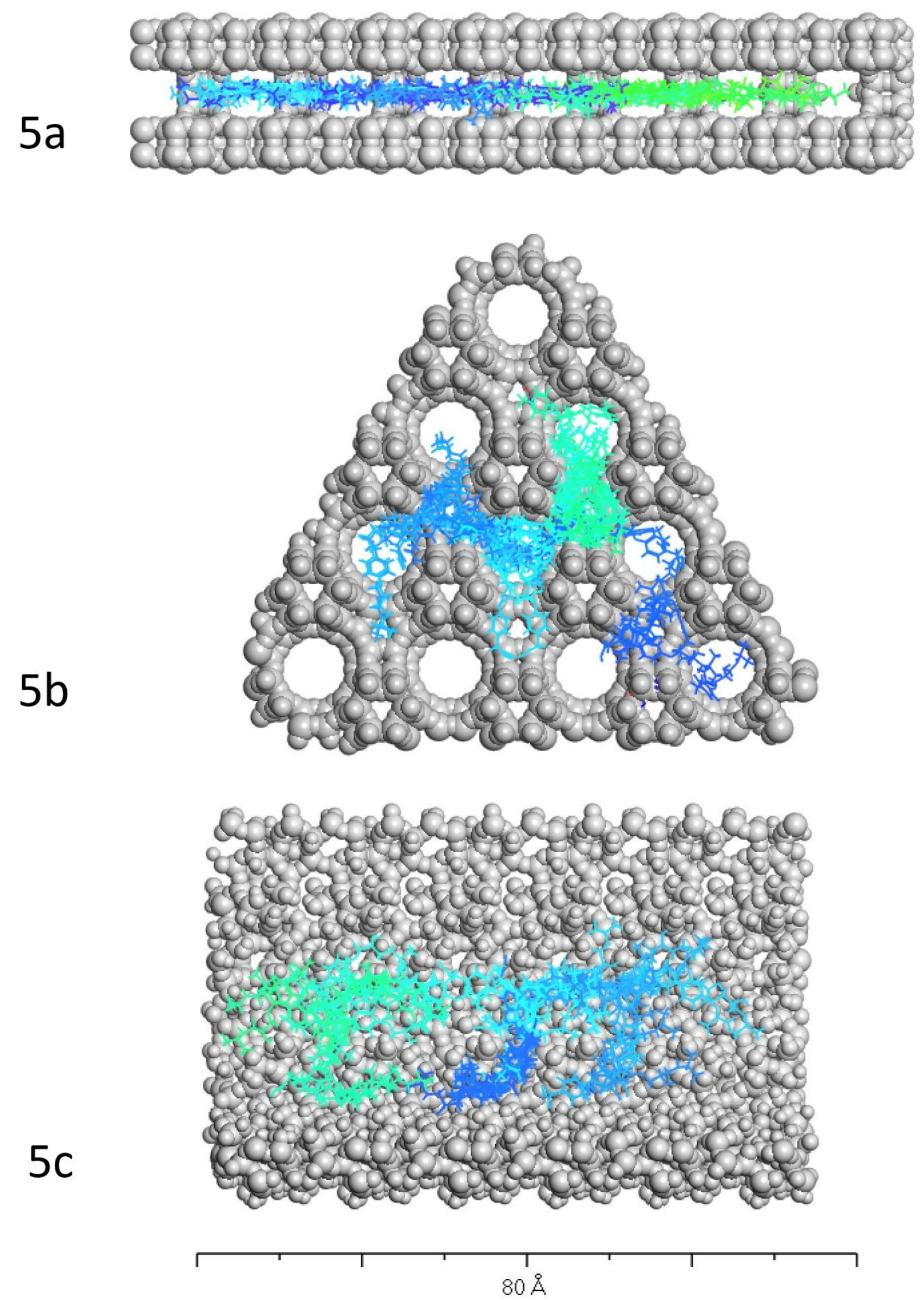

Figure 4 Figures 4 a-c show the Location of Atenolol Every 400 ps During MD Calculations in b. BEA, c. FAU and d. MCM-41 with the initial location in dark green blending to the final location in dark blue. 


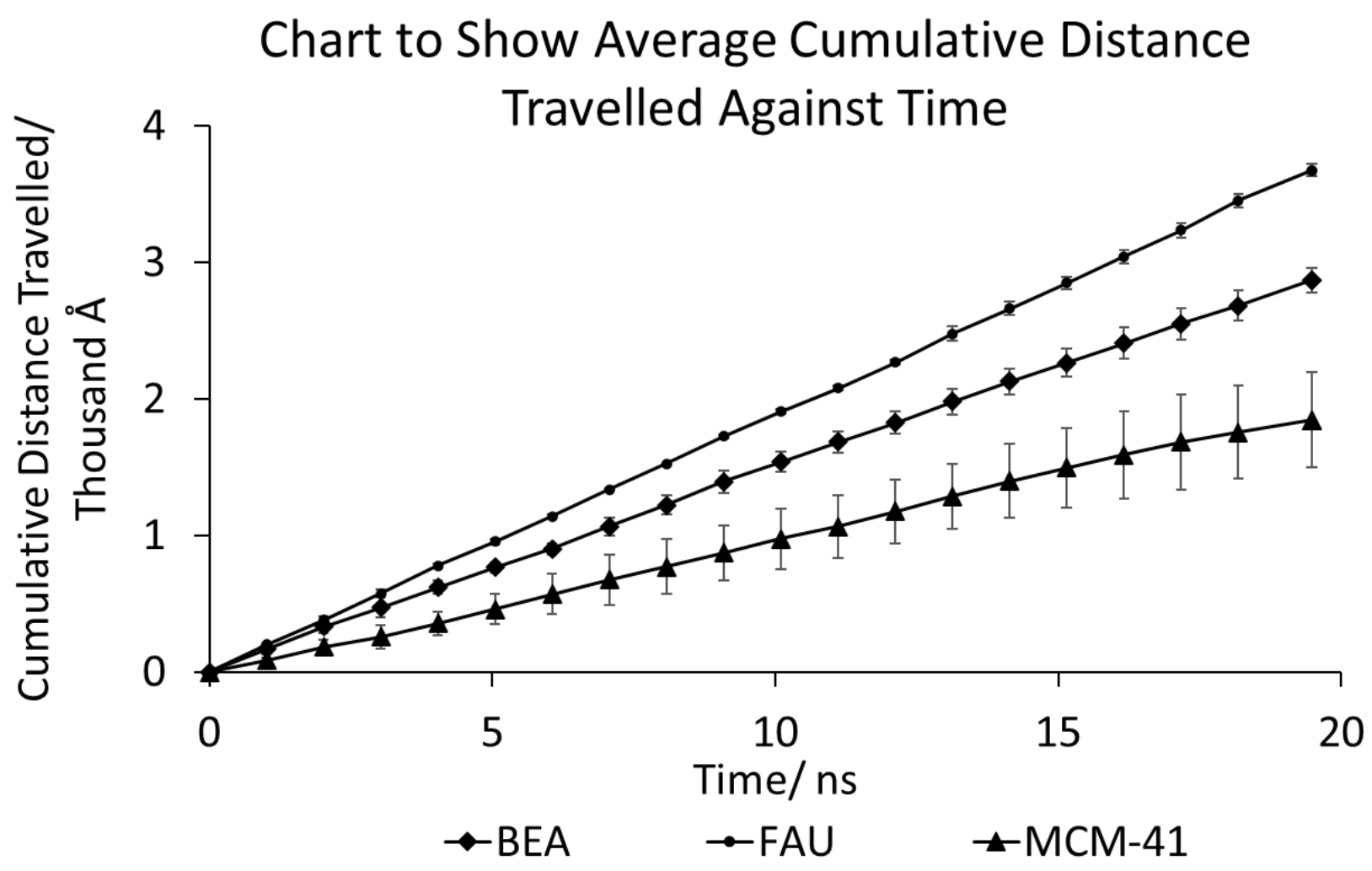

Figure 5 The average distance travelled against time for the atenolol centroid during the molecular dynamics simulations. Data displayed is every $100^{\text {th }}$ data point to allow error bars to be displayed. Error bars shown are standard deviation.

Figures 4a-c illustrate the movement of the drug molecules during the molecular dynamics simulations, while figure 5 shows the distance travelled by the centroid of the atenolol molecule against time from MD simulations. Distance travelled is presented here in preference to RMS displacement data as the atenolol molecule is often observed to reverse direction along a channel during the course of the simulations. This effect is particularly noticeable in these simulations because of the long simulation time and slow movement of the drug within the host lattices. It is particularly the case for the drug molecule within the BEA framework, where the atenolol molecule can spend a significant amount of time at a channel intersection before 'hopping' to the next channel intersection. In the simulations, there is no driving force for the molecule to move preferentially in one direction along the channel, unlike the experiments where the external media will exert an influence on the direction of travel of the molecule.

It is apparent from the illustrations that the movement of the drug molecule is very different within each of the frameworks while the simulation data show that the diffusion of the drug molecule is dependent on the framework into which it is loaded, movement being much faster in FAU than BEA or MCM-41. In the BEA framework, the movement of the drug molecule is limited to travel along one plane because, due to steric hindrance, it is not possible for the drug molecule to move from one channel to another adjacent channel via the intersections and accordingly the drug molecule is confined to the channel it is placed in at the start of the simulation. The drug molecule also appears to remain resident at channel intersections for prolonged periods of time before 'hopping' to another adjacent channel intersection.

The atenolol molecule in the FAU framework simulation demonstrates very different movement to that seen in BEA. Unlike the simulation in BEA, the atenolol molecule is able to bend, twist and move freely in all directions within the supercages. It remains in a cage for a period of time until it is suitably aligned to travel through the interlinking window into an adjacent supercage. 
The simulation using the MCM-41 framework demonstrates that surface interaction between the mesopore surface and drug molecule presents a significant barrier to drug molecule movement rather than steric hindrance due to channel dimensions. It is clear from the MCM-41 simulation data that, despite the absence of steric hindrance, the movement of the drug molecule is slower than for the FAU and BEA frameworks. Since the simulation data are reproducible, have narrow standard deviation values, and show that atenolol moves faster in FAU than in BEA, the suggested use of $\mathrm{MD}$ as a screening tool for predicting effect of steric hindrance and thus release rate on drug-zeolite combinations is supported, but the current model does not appear to allow effective comparisons of release rate to be made between the microporous and mesoporous structures.

Figure 6 presents a comparison between the average distance travelled for the centroid of the atenolol molecule within the two zeolite frameworks when the atenolol molecule is protonated, with the appropriate adjustment to the charge of the framework made, as outlined in the method section. It can be seen that the effects of protonating the molecule are relatively small, with only a small change in the rate of movement of the drug molecule inside the FAU framework. A more pronounced effect in observed for BEA, but the rate of movement in this framework is still significantly less than in FAU. The mechanism of movement in the two frameworks remains the same.

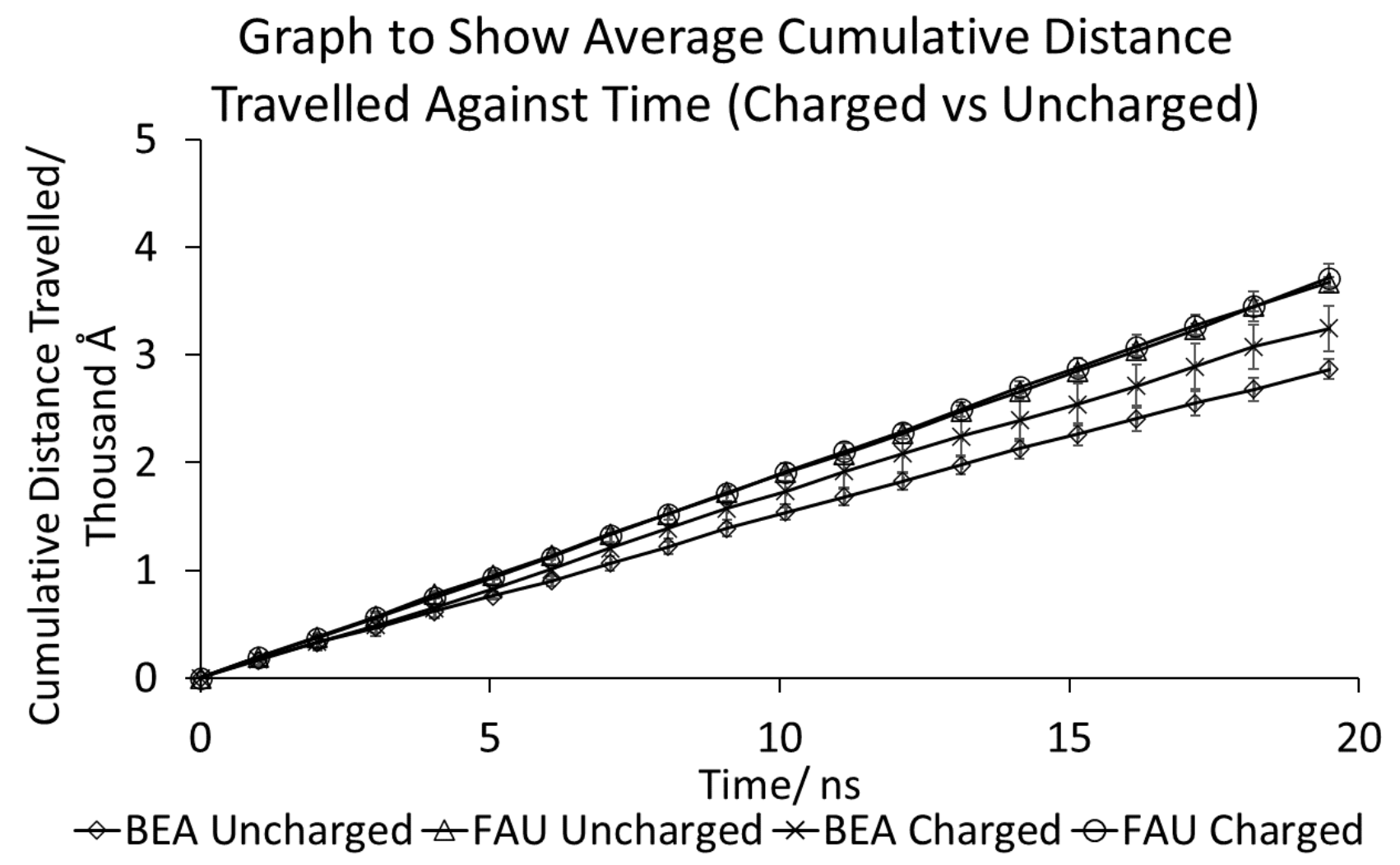

Figure 6: Graph to Show Average Cumulative Distance Travelled for Uncharged Atenolol in Uncharged BEA (diamond) and FAU(triangle) frameworks and +1 Charged Atenolol in -1 Charged BEA (cross) and FAU (circle) Frameworks 


\section{Discussion}

\section{Effect of Framework Topology}

It is apparent that the choice of framework can have a significant impact on both loading and release of atenolol, and this may in part be explained by the effect of steric hindrance on diffusion of the drug molecule.

Steric hindrance of the drug due to limited pore and channel sizes is likely to be greatest in BEA, where the largest diameter of a sphere that can diffuse along the channels is just $5.95 \AA$ (16) and there is a relatively tight fit of the atenolol molecule with limited movement allowed. The FAU framework will also cause steric hindrance, but this will be less because the maximum sphere size that can diffuse through the framework is significantly higher at $7.35 \AA$ (16). Importantly, though, the atenolol molecule has more room to bend and rotate in FAU than in BEA, particularly within the super cages, and thus the effect of steric hindrance should be greater within BEA. These observations agree with the experimental data which show that BEA has the lowest loading and lowest relative quantity released of all the frameworks into both acid and buffer. The experimental and simulation data for drug release rates also agree with this, since drug release in experimental data and drug diffusion in simulation data is slower in BEA than in FAU.

In contrast, the mesoporous materials have much wider pore diameters $(21-30 \AA)(19,20)$ which are wider than the maximum diameter of the atenolol molecule, and steric hindrance would be unlikely to be a significant barrier to drug diffusion. It might follow then that drug loading should be greater for all mesoporous materials than for zeolites. However, as the materials have been loaded with only a small mass of drug, it is unclear if the frameworks in this study are saturated and is therefore not possible to determine if the mesoporous frameworks can load greater quantities of atenolol than zeolites. Further investigations are needed to establish whether different experimental conditions can result in greater drug loading and determine what mass of drug is loaded when these frameworks are saturated.

If steric hindrance was the only barrier to drug diffusion, it would also be expected that drug release would be fastest from the MCM-41 materials. However, it is apparent that release from MCM-41 is similar in buffer and slower in acid when compared to release from FAU 5.1:1. This implies that, while steric hindrance is a factor in drug release rate, other contributory factors are also significant, discussed next.

\section{Effect of Aluminium Content}

In addition to the effect of framework topology, it is also notable that aluminium content can have a significant effect on loading and release from both zeolite and MCM-41 frameworks.

In the zeolite structures used in this study, $\mathrm{H}^{+}$ions counter the negative charge introduced to the framework by the replacement of silicon atoms by aluminium atoms as the $\mathrm{SiO}_{2} / \mathrm{Al}_{2} \mathrm{O}$ ratio is reduced. These $\mathrm{H}^{+}$ions lead to the formation of $\mathrm{Br} ø$ nsted acid sites with the $\mathrm{H}^{+}$ions bound to framework oxygen atoms adjacent to aluminium.

The creation of additional Brønsted acid sites as the $\mathrm{SiO}_{2} / \mathrm{Al}_{2} \mathrm{O}$ ratio decreases could, in theory, have the potential to either increase or decrease the interaction between the zeolite framework and the atenolol molecules. Increased interaction could result from the formation of hydrogen bonds between the drug and the framework. However, this would result in slower release of the atenolol as the $\mathrm{SiO}_{2} / \mathrm{Al}_{2} \mathrm{O}$ ratio is reduced, not the increased rate that is observed in the experiments. Instead, it seems that the additional $\mathrm{H}^{+}$ions reduce the electrostatic interaction between the framework and the 
atenolol molecules. This seems particularly plausible as the atenolol molecule, with a pK $\mathrm{K}_{\mathrm{a}}$ of 9.6 (25), will be protonated at both $\mathrm{pH}=1$ and 7.4 resulting in electrostatic repulsion from the framework. The distribution of the Brønsted acid sites in the framework may also be an important factor. Furthermore, lower levels of $\mathrm{H}^{+}$ions at higher $\mathrm{SiO}_{2} / \mathrm{Al}_{2} \mathrm{O}$ ratios may enhance the probability of favourable interactions between the drug and Brønsted acid sites, where the $\mathrm{H}^{+}$ions are more spread out along the channels, although the distribution of these sites is a complex issue as aluminium nests are known to exist where $\mathrm{SiO}_{2} / \mathrm{Al}_{2} \mathrm{O}$ ratios are high $(26,27)$.

It should be noted that solvent effects may also provide an explanation for the observed differences in drug release at different $\mathrm{SiO}_{2} / \mathrm{Al}_{2} \mathrm{O}$ ratios. Increased wettability of frameworks with lower $\mathrm{SiO}_{2}: \mathrm{Al}_{2} \mathrm{O}_{3}$ ratios(3), means that the sites available for hydrogen bonding may be occupied by water molecules. The importance of water molecules at the silica surface has been reported to be of significance in MCM-41 loaded with ibuprofen and the authors suggest that this is a consideration that is frequently overlooked when investigating drug delivery from silica(28). In addition, there is evidence that Brønsted Acid Sites can have mutual interaction via hydrogen bonding and, as a result, greater numbers of acid sites do not correlate with greater acidity(29). A combination of these factors may result in hydrogen bonding being of a similar strength in frameworks with the same topology regardless of the $\mathrm{SiO}_{2}: \mathrm{Al}_{2} \mathrm{O}_{3}$ ratio.

\section{Effect of $\mathrm{pH}$}

Lastly, the effect of $\mathrm{pH}$ on drug release rate is demonstrated. In general, the release of atenolol is slower into phosphate buffer than into acid. This implies that the strength of the drug-framework interactions present is dependent on the $\mathrm{pH}$ and may be due to $\mathrm{pH}$ affecting the charge of the framework, the drug molecule, water molecules, or a combination of all of these.

The log $\mathrm{P}$ value of atenolol is 0.43 , however at $\mathrm{pH} 1.2$ the $\log \mathrm{D}$ value is -2.817 while at $\mathrm{pH} 7.4$ it is 1.800 (30) and is therefore protonated at both $\mathrm{pH}$ values. As the $\mathrm{pH}$ increases, the $\mathrm{Br} \varnothing$ nsted acid sites in the zeolite are more likely to be deprotonated (31). This will lead to a reduction in the electrostatic repulsion between the $\mathrm{H}^{+}$framework ions and the protonated drug molecules. Hence, faster release is observed in a lower pH environment. Similarly, the charge on the MCM-41 internal surface will be more positive in an acid environment, leading to greater electrostatic repulsion between the drug and the framework. It is interesting to note the release from the FAU 5.1:1 sample is much more pHdependent than the other samples. This can be attributed to its higher aluminium content

It is important to recognise that, given the flattening of the curves in the drug release studies before achieving $100 \%$ drug release, it is unlikely that all of the drug will be released from the samples. This indicates that, while electrostatic repulsion may be a key factor in determining the release rate, there must be other drug-framework interactions taking place that are strong enough to prevent release of a proportion of the drug molecules. Other authors have suggested possible interactions including weak intermolecular hydrogen bonds between silanol groups and drug molecules $(32,33)$, coordination complexation (32), and van der Waals forces $(33,34)$. The complex nature of the drugframework interactions further highlight the benefits of developing MD simulations and other modelling techniques to help understand the relative importance of all these competing factors. 


\section{Molecular Dynamics}

The simulations presented in this work show that MD can be used as a useful predictor of steric hindrance for drug-framework combinations, with movement of the drug molecule within the FAU structure much faster than BEA. This suggests that they can usefully be deployed in order to screen suitable framework types for prolonged release formulations. MD simulations also yield a unique insight into the mechanism of movement of the drug inside the internal channel systems.

As demonstrated by the experimental data, the presence and strength of drug-framework interactions are strongly influenced by both aluminium content and $\mathrm{pH}$. This emphasises the importance of charge in these types of calculation. The atenolol molecule is protonated, which requires appropriate adjustment of the charge of the framework as periodic molecular dynamics simulations require a net charge of zero within the simulation box. Explicit inclusion of counter ions, such as $\mathrm{H}^{+}$will be important in order to use these techniques to predict the influence of $\mathrm{SiO}_{2} / \mathrm{Al}_{2} \mathrm{O}$ ratios on release rates.

The simulations reported here do not have any solvent molecules in the system. This could help to explain why the MD data show atenolol moving slower in MCM-41 than the two zeolite framework types. Explicit Inclusion of solvent molecules is likely to be important in release rates, particularly in the case of mesoporous materials where the pore volumes are relatively high. The absence of water molecules might also explain why the data show the rate of movement in BEA to be much more similar to FAU in MD simulations when compared to lab experiments. This is because, in addition to the drugframework interactions that would be occurring, the presence of water may increase the effect of steric hindrance, particularly in BEA where there is already a tight fit in the framework channel. Including water molecules is feasible, but will increase the computational time significantly, particularly in the case of mesoporous materials.

Only one molecule per simulation box has been included in the simulations reported here. Again, this could be an important limitation, particularly in the case of mesoporous materials where the increased pore size means that drug-drug interactions are likely to be more significant.

The development of simulations which account for all factors that affect drug release rates would be extremely desirable since the effect of framework aluminium content and $\mathrm{pH}$ are very difficult to predict. Appropriate models will need to explicitly include aluminium atoms, appropriate charge balancing cations, such as $\mathrm{H}^{+}$and solvent molecules in order to help understand the effects of these factors on release rates. 


\section{Conclusions}

The results presented in this study illustrate the potential of using microporous and mesoporous silica for the prolonged release of atenolol. This was most notable with FAU 80:1 which was able to provide zero order drug release for over $24 \mathrm{hr}$ into phosphate buffer (used to simulate the conditions of intestinal fluid), and continued to slowly release atenolol for a further $48 \mathrm{hr}$. It was unexpected that this particular combination of zeolite and drug would give rise to such a remarkable release profile and hence a screening tool that can identify specific drug-framework combinations that give rise to specific release profiles is highly desirable.

Along with previous studies $(13,14)$, the potential for MD as a screening tool to predict the effect of steric hindrance in drug-zeolite combinations is again demonstrated, and it is proposed that MD represents a suitable pre-screen for identifying suitable frameworks for delayed release. However, this study highlights that sophisticated models are required in order to predict the effects of changes such as $\mathrm{SiO}_{2} / \mathrm{Al}_{2} \mathrm{O}$ ratios or $\mathrm{pH}$. Including water molecules in the system and explicitly including aluminium and counter-ions will be needed to simulate drug-framework interactions more accurately so that predicting the effects of these subtle changes can be achieved prior to carrying out lab based experiments. 


\section{References}

1. Servatan M, Zarrintaj P, Mahmodi G, Kim S-J, Ganjali MR, Saeb MR, Mozafari M. Zeolites in drug delivery : Progress, challenges and opportunities, Drug Discov Today, 25(4), p.642-56, 2020.

2. Mintova S, Jaber M, Valtchev V. Nanosized microporous crystals: emerging applications. Chem Soc Rev. 44(20), 2015.

3. Vallet-Regí M, Balas F, Arcos D. Mesoporous Materials for Drug Delivery. Angew Chemie Int Ed. 46(40):7548-58, 2007.

4. Kwon S, Singh RK, Perez RA, Abou Neel EA, Kim H-W, Chrzanowski W. Silica-based mesoporous nanoparticles for controlled drug delivery. J Tissue Eng [Internet]. 4:2041731413503357, 2013.

5. Tavolaro A, Riccio II, Tavolaro P. Hydrothermal synthesis of zeolite composite membranes and crystals as potential vectors for drug-delivering biomaterials. Micropor Mesopor Mat. 167, p62-70 (2013).

6. Berger D, Nastase S, Mitran RA, Petrescu M, Vasile E, Matei C, et al. Mesostructured silica and aluminosilicate carriers for oxytetracycline delivery systems. Int J Pharm. 510(2), p. 524-31, 2016.

7. Horcajada P, Márquez-Alvarez C, Rámila A, Pérez-Pariente J, Vallet-Regí M. Controlled release of Ibuprofen from dealuminated faujasites. Solid State Sci, 8(12), p.1459-65, 2006.

8. Eriksson $\mathrm{H}$. Controlled release of preservatives using dealuminated zeolite Y. J Biochem Biophys Methods, 70(6), p.1139-44, 2008.

9. Zaarour M, Dong B, Naydenova I, Retoux R, Mintova S. Progress in zeolite synthesis promotes advanced applications. Micropor Mesopor Mat, 189, p.11-21, 2018.

10. Karavasili C, Kokove L, Kontopoulou I, Eleftheriadis GK, Bouropoulos N, Fatouros DG. Dissolution enhancement of the poorly soluble drug nifedipine by co-spray drying with microporous zeolite beta. J Drug Deliv Sci Technol ,35, p.91-97, 2016.

11. Baerlocher C, McCusker L B HRM. Database of Zeolite Structures [Internet]. [cited 2020 Jan 22]. Available from: http://www.iza-structure.org/databases/

12. Salonen J, Kaukonen AM, Hirvonen J, Lehto V-P. Mesoporous silicon in drug delivery applications. J Pharm Sci, 97(2), p.632-53, 2008.

13. Mohammadzadeh M, Nourbakhsh MS, Khodaverdi E, Hadizadeh F, Omid Malayeri S. Enhanced Loading and Release of Non-Steroidal Anti-Inflammatory Drugs from Silica-Based Nanoparticle Carriers. Chem Biol Drug Des [Internet], 88(3), p.370-9, 2016.

14. Spanakis M, Bouropoulos N, Theodoropoulos D, Sygellou L, Ewart S, Moschovi AM, et al. Controlled release of 5-fluorouracil from microporous zeolites. Nanomedicine Nanotechnology, Biol Med, 10(1), p.197-205, 2014.

15. Fatouros DG, Douroumis D, Nikolakis V, Ntais S, Moschovi AM, Trivedi V, et al. In vitro and in silico investigations of drug delivery via zeolite BEA. J Mater Chem, 21(21), p.7789-7794, 2011.

16. 1318-02-1 - Zeolite beta, hydrogen - 45875 - Alfa Aesar [Internet]. [cited 2020 Jan 27]. 
Available from: https://www.alfa.com/en/catalog/045875/

17. 1318-02-1 - Zeolite Y, hydrogen - Faujasite - 45866 - Alfa Aesar [Internet]. [cited 2020 Jan 27]. Available from: https://www.alfa.com/en/catalog/045866/

18. 1318-02-1 - Zeolite Y, hydrogen - Faujasite - 45872 - Alfa Aesar [Internet]. [cited 2020 Jan 27]. Available from: https://www.alfa.com/en/catalog/045872/

19. Silica, mesostructured MCM-41 type (hexagonal) | Sigma-Aldrich [Internet]. [cited 2020 Jan 22]. Available from:

https://www.sigmaaldrich.com/catalog/product/aldrich/643645?lang=en\&region=GB

20. Aluminosilicate, mesostructured MCM-41 (hexagonal) | Sigma-Aldrich [Internet]. [cited 2020 Jan 22]. Available from:

https://www.sigmaaldrich.com/catalog/product/aldrich/643653?lang=en\&region=GB

21. BIOVIA, Dassault Systèmes, Materials Studio 2019, San Diego: Dassault Systèmes, 2019.

22. Ugliengo P, So.dupe M, Musso F, Bush I, Orlando R, Dovesi R. Realistic models of hydroxylated amorphous silica surfaces and MCM- 41 mesoporous materials simulatied by large-scale periodic B3IYP calculations, Adv Mater., 20(23), 4579-4583, 2008.

23. Turrina A, Garcia R, Watts AE, Greer HF, Bradley J, Zhou W, et al., STA-20: An ABC-6 zeotype structure prepared by co-templating and solved via a hypothetical structure database and STEM-ADF imaging. Chem Mater., 29(5), p.2180-2190, 2017.

24. Castaldi P, Santona L, Cozza C, Giuliano V, Abbruzzese C, Nastro V, et al. Thermal and spectroscopic studies of zeolites exchanged with metal cations. J Mol Struct, 734(1-3), p.99105, 2005.

25. National Center for Biotechnology Information. PubChem Database. Atenolol, CID=2249, https://pubchem.ncbi.nlm.nih.gov/compound/Atenolol (accessed on May 25, 2020).

26. Lutz W. Zeolite Y: Synthesis, modification, and properties - A case revisited, Adv Mater Sci Eng, 2014, p1-20 .

27. Van Bokhoven JA, Koningsberger DC, Kunkeler P, Van Bekkum H, Kentgens APM. Stepwise dealumination of zeolite Beta at specific T-sites observed with 27AI MAS and 27AI MQ MAS NMR. J Am Chem Soc, 122(51), p.12842-7, 2000.

28. Azaïs T, Laurent G, Panesar K, Nossov A, Guenneau F, Sanfeliu Cano C, et al. Implication of Water Molecules at the Silica-Ibuprofen Interface in Silica-Based Drug Delivery Systems Obtained through Incipient Wetness Impregnation. J Phys Chem C., 121(48), p.26833-26839, 2017.

29. Busca G. Acidity and basicity of zeolites: A fundamental approach. Micropor Mesopor Mat, 254, p.3-16, 2017.

30. Chemicalize [Internet]. [cited 2020 Jan 22]. Available from: https://chemicalize.com/\#/calculation

31. Kasmi T, Soualah A, Mignard S, Batonneau-Gener I. Effect of Bronsted aciditiy of HY zeolites in adsoprtion of methylene blue and comparative study with bentonite. J Environ Health Sci., 16(2), p.239-247, 2018.

32. Song S-W, Hidajat $\mathrm{K}$ and Kawi S. Functionalized SBA-15 materials as carriers for controlled drug delivery: Influence of surface properties on matrix-drug interactions. Langmuir, 21(21), p.9568-9575, 2005. 
33. Datt A, Fields D and Larsen SC. An experimental and computational study of the loading and release of aspirin from zeolite HY. J Phys Chem C, 116(40), p21382-23190, 2012.

34. Datt A, Burns EA, Dhuna NA and Larsen SC. Loading and release of 5-fluorouracil from HY zeolites with varying $\mathrm{SiO}_{2} / \mathrm{Al}_{2} \mathrm{O}_{3}$ ratios. Micropor Mesoor Mat, 167, p.182-187, 2013. 